\title{
Fast approximate radiative transfer method for visualizing the fine structure of prominences in the hydrogen $\mathrm{H} \alpha$ line
}

\author{
P. Heinzel ${ }^{1,3}$, S. Gunár ${ }^{2, \dagger}$, and U. Anzer ${ }^{3}$ \\ 1 Astronomical Institute, The Czech Academy of Sciences, Czech Republic, 25165 Ondřejov, Czech Republic \\ e-mail: pheinzel@asu.cas.cz \\ 2 School of Mathematics and Statistics, University of St Andrews, North Haugh, St Andrews, KY16 9SS, UK \\ ${ }^{3}$ Max-Planck-Institut für Astrophysik, Karl-Schwarzschild-Str. 1, 85740 Garching bei München, Germany
}

Received 22 January 2015 / Accepted 9 April 2015

\begin{abstract}
Aims. We present a novel approximate radiative transfer method developed to visualize 3D whole-prominence models with multiple fine structures using the hydrogen $\mathrm{H} \alpha$ spectral line.

Methods. This method employs a fast line-of-sight synthesis of the $\mathrm{H} \alpha$ line profiles through the whole 3D prominence volume and realistically reflects the basic properties of the $\mathrm{H} \alpha$ line formation in the cool and low-density prominence medium. The method can be applied both to prominences seen above the limb and filaments seen against the disk.

Results. We provide recipes for the use of this method for visualizing the prominence or filament models that have multiple fine structures. We also perform tests of the method that demonstrate its accuracy under prominence conditions.

Conclusions. We demonstrate that this fast approximate radiative transfer method provides realistic synthetic $\mathrm{H} \alpha$ intensities useful for a reliable visualization of prominences and filaments. Such synthetic high-resolution images of modeled prominences/filaments can be used for a direct comparison with high-resolution observations.
\end{abstract}

Key words. radiative transfer - Sun: filaments, prominences - Sun: magnetic fields

\section{Introduction}

During the past two decades, a significant effort was devoted to 3D modeling of prominence magnetic structures - see, for example, the reviews by Mackay et al. (2010), van Ballegooijen \& Su (2014), and Gunár (2014). For quiescent prominences, a variety of magnetic field configurations with numerous magnetic dips was produced with these models. These magnetic dips are routinely visualized schematically by plotting their characteristic length, often corresponding to one pressure scaleheight $(300 \mathrm{~km}$ if assuming an isothermal plasma with a temperature of $10000 \mathrm{~K}$ ), see, for instance, Aulanier \& Démoulin (1998), Dudík et al. (2008), Mackay \& van Ballegooijen (2009), and $\mathrm{Su} \&$ van Ballegooijen (2012). Such illustrative visualization provides an idea about the global pattern of the prominence or filament structures. However, the magnetic field configurations visualized in this way cannot be directly compared with high-resolution images of the prominence or filament fine structures obtained by various instruments, often in the $\mathrm{H} \alpha$ line. The main reason is that the prominence $\mathrm{H} \alpha$ visibility largely depends on the number of fine structures projected into the line of sight (LOS). And while the $\mathrm{H} \alpha$ optical thickness of individual prominence fine structures may be significantly below unity, the total optical thickness along the LOS in typical quiescent prominences is larger. The effects of radiative transfer along the LOS therefore have to be taken into account to provide more realistic prominence visualizations.

$\dagger$ On leave from Astronomical Institute of the Czech Academy of Sciences.
To visualize a prominence consisting of many dips, first individual magnetic dips must be filled by the plasma with realistic temperature and gas pressure distributions and then, ideally, one has to perform 3D radiative-transfer modeling of such highly heterogeneous prominence to predict its visibility in a given spectral line. One way to fill the dips with prominence plasma is to consider the thermal non-equilibrium models of Karpen et al. (2006), which are based on the heating and evaporation of the chromospheric plasma and its subsequent cooling and condensation inside the magnetic dips. Luna et al. (2012) have shown that these prominence formation models can predict the signatures of hotter parts of the prominence fine structures (such as thermal properties, velocities, and mass loading) but do not directly provide the $\mathrm{H} \alpha$ visibility of the cool fine structures. Recently, Gunár et al. (2013) developed a method allowing to fill individual force-free magnetic dips with the prominence plasma in a hydrostatic equilibrium. These dips can be approximated by 2D vertically infinite threads similar to those of Heinzel \& Anzer (2001), and thus the 2D non-LTE (i.e., departures from local thermodynamic equilibrium) radiative transfer modeling can be used to obtain their radiative output in hydrogen spectral lines. Gunár \& Mackay (2015) used the method of filling the force-free dips with prominence plasma to construct the first 3D whole-prominence fine structure (WPFS) models. These are based on the nonlinear forcefree field simulations of the entire prominence magnetic field (Mackay \& van Ballegooijen 2009) and contain realistic prominence plasma distributed along a large number of fine structures. However, an implementation of the full 3D non-LTE computations to such extensive highly heterogeneous models with 
hundreds of prominence fine structures would present a task that is extremely demanding on computing resources. This is dictated by the need to use fine grid-spacing down to a few $\mathrm{km}$ to properly resolve steep temperature gradients within individual plasma fine structures. Such fine grid-spacing is also required to accurately solve the radiative transfer in the optically thick lines such as the Lyman- $\alpha$ line of hydrogen. Moreover, the structure of entire prominences can span large dimensions on the order of $200000 \times 50000 \times 20000 \mathrm{~km}$ (Gunár \& Mackay 2015). This would lead to extremely large computational grids, and even with adaptive mesh refinement such computations would still significantly exceed the computing requirements of current massive radiation-magneto-hydrodynamic simulations such as the 3D chromospheric simulations of Leenaarts et al. (2012) and Leenaarts et al. (2015).

In this paper we present a fast approximate method for visualizing 3D whole-prominence models in the $\mathrm{H} \alpha$ line that can be used for imaging of the cool prominence structures. This method is based on our experience with the $\mathrm{H} \alpha$ line formation in prominences, see, for example, Heinzel (2015). It employs a fast line-of-sight synthesis of the $\mathrm{H} \alpha$ line profiles through the whole 3D prominence volume and realistically reflects the basic properties of the $\mathrm{H} \alpha$ line formation in the cool and lowdensity prominence medium. The method can be applied both to prominences seen above the limb and to filaments seen against the disk. It was recently used by Gunár \& Mackay (2015) to visualize their 3D WPFS model.

We present the $\mathrm{H} \alpha$ visualization method in Sect. 2 and give recipes for its use in Sect. 3. Section 3 also provides the results of several tests comparing $1 \mathrm{D}$ and $2 \mathrm{D}$ non-LTE radiative transfer models and the new fast visualization method. In Sect. 4 we present a sample image of visualized force-free magnetic dips, and in Sect. 5 we discuss our results and make conclusions.

\section{H $\alpha$ synthesis}

The H $\alpha$ intensity is obtained by the LOS integration of the radiative transfer equation through generally nonuniform prominence media. To obtain the $\mathrm{H} \alpha$ intensity, we must know the distribution of the line source function and the opacity along the LOS. The specific intensity is then obtained from the formal solution of the radiative transfer equation

$I(v)=\int_{0}^{\tau_{v}} S\left(t_{v}\right) \mathrm{e}^{-t_{v}} \mathrm{~d} t_{v}$,

where

$\mathrm{d} t_{v}=\kappa(v, l) \mathrm{d} l$.

The term $\mathrm{d} t_{v}$ represents the optical-depth increment corresponding to the geometrical path increment $\mathrm{d} l$, and $\kappa(v, l)$ is the absorption coefficient for the $\mathrm{H} \alpha$ line. $\tau_{v}$ is the total optical thickness integrated through all structures crossed by a given LOS. Assuming a uniform source function in the whole medium, Eq. (1) reduces to the well-known from (see, e.g., Heinzel 2015)

$I(v)=S\left(1-\mathrm{e}^{-\tau_{v}}\right)$.

The absorption coefficient for the $\mathrm{H} \alpha$ line has the standard form

$\kappa(v, l)=\frac{\pi e^{2}}{m_{\mathrm{e}} c} f_{23} n_{2} \phi(v)=1.7 \times 10^{-2} n_{2} \phi(v)$,

where $f_{23}$ is the $\mathrm{H} \alpha$ line oscillator strength, $n_{2}$ is the hydrogen second-level population, and $\phi(v)$ is the normalized absorption profile. Since the individual fine structures are assumed to be optically thin (see below), the absorption line profile is well approximated by a Gaussian profile in our case, depending on the thermal and microturbulent broadening

$\phi(v)=\frac{1}{\sqrt{\pi} \Delta v_{\mathrm{D}}} \exp \left(-\frac{\Delta v}{\Delta \nu_{\mathrm{D}}}\right)^{2}$,

where

$\Delta v_{\mathrm{D}}=\frac{v_{0}}{c}\left(\frac{2 k T}{m_{\mathrm{H}}}+\xi_{\mathrm{t}}^{2}\right)^{1 / 2}$.

We assume a given LOS distribution of the kinetic temperature $T$ and gas pressure $p_{\mathrm{g}}$. The microturbulent velocity is difficult to determine and thus we take a representative value of $5 \mathrm{~km} \mathrm{~s}^{-1}$ in the whole prominence (see, e.g., Engvold et al. 1990; Labrosse et al. 2010). Certain variations would not affect the $\mathrm{H} \alpha$ prominence visibility significantly. To compute the line intensity according to Eq. (1), we need to know the value of the source function and the LOS variations of the hydrogen second-level population, which enters the absorption coefficient, see Eq. (4).

\subsection{Ho line source function}

Under typical prominence conditions, the $\mathrm{H} \alpha$ line source function is dominated by the scattering of the incident radiation from the solar disk (Heinzel 2015), which depends on the altitude of the prominence material above the solar surface. For an altitude of $10000 \mathrm{~km}$, Gouttebroze et al. (1993) constructed a grid of 140 isothermal-isobaric 1D slab models and tabulated various structural and radiative quantities. Based on this, Heinzel et al. (1994) demonstrated several important correlations between these quantities. One of the most important results is that the $\mathrm{H} \alpha$ source function is nearly constant in 1D slabs for the line-center optical thicknesses up to unity. Above this value, the $\mathrm{H} \alpha$ source function starts to increase (see Fig. 18 in Heinzel et al. 1994). At an altitude of $10000 \mathrm{~km}$ the source function $S$ is fixed at a value approximately equal to the diluted $\mathrm{H} \alpha$ line-center intensity at the solar disk center, which is $W \times 0.17 \times 4.077 \times 10^{-5} \mathrm{erg} \mathrm{s}^{-1} \mathrm{~cm}^{-2} \mathrm{sr}^{-1} \mathrm{~Hz}^{-1}$. The dilution factor $W$ has the form

$W=\frac{1}{2}\left(1-\sqrt{1-\frac{R^{2}}{(R+H)^{2}}}\right)$,

where $H$ represents the height above the solar surface and $R$ is the solar radius (see, e.g., the Appendix in Jejčič \& Heinzel 2009). The factor 0.17 corresponds to the central depression of the $\mathrm{H} \alpha$ line at the disk center (David 1961) and $4.077 \times 10^{-5} \mathrm{erg} \mathrm{s}^{-1} \mathrm{~cm}^{-2} \mathrm{sr}^{-1} \mathrm{~Hz}^{-1}$ is the disk-center continuum intensity near the $\mathrm{H} \alpha$ line.

The prominence fine structures are typically small and numerous along a given LOS. While the total $\mathrm{H} \alpha$ optical thickness of large prominences may reach values higher than one, we therefore assume that each fine structure element (magnetic dip filled with the prominence plasma) is optically thin in $\mathrm{H} \alpha$. This allows us to use a uniform value of the line source function at the given altitude. For different altitudes this value scales roughly with the ratio of the dilution factors (see Sect. 2.3). Note also that the $\mathrm{H} \alpha$ line source function is frequency-independent in the CRD (complete frequency redistribution) approximation. Specifying the $\mathrm{H} \alpha$ line source function in this way, we assumed that all structures are independently illuminated by the solar disk 
radiation and that they do not interact radiatively among themselves. However, the $\mathrm{H} \alpha$ source function will remain similar even for more densely packed structures.

\subsection{H $\alpha$ absorption coefficient}

The only unknown quantity in Eq. (4) is the population (number density) of hydrogen atoms in the second quantum level $n_{2}$. To determine this in an approximate way, we use the relation between the electron density and the hydrogen second-level population

$n_{\mathrm{e}}^{2}=f\left(T, p_{\mathrm{g}}\right) n_{2}$.

This relation was derived by various authors, and its nature is explained in Heinzel et al. (1994), who found $f \simeq 3 \times 10^{16} \mathrm{~cm}^{-3}$ for $T=8000 \mathrm{~K}$. The same value was also reported by Landman \& Mongillo (1979). In general, this factor mildly depends on the temperature and gas pressure (see Table 1). The electron density can be estimated from the local values of the gas pressure and the temperature using the following set of formulas:

$p_{\mathrm{g}}=N k T$,

where $N$ is the total particle number density given by $N=n_{\mathrm{H}}+$ $n_{\mathrm{He}}+n_{\mathrm{e}}$. Here, $n_{\mathrm{H}}$ is the total hydrogen density - the sum of the neutral hydrogen and the proton density, and $n_{\mathrm{He}}$ is the helium density. Assuming 10\% abundance of the helium and no helium ionization (fully ionized helium would contribute to the electron density by $20 \%$ ), we can write

$p_{\mathrm{g}}=\left(1+\frac{1.1}{i}\right) n_{\mathrm{e}} k T$

where $i=n_{\mathrm{e}} / n_{\mathrm{H}}$ is the hydrogen ionization degree. The value of $i$ depends on temperature and gas pressure and is specified below.

\subsection{Parameters $S, f$, and $i$}

Mean values of $i$ and $f$ can be inferred from the correlation plots of Heinzel et al. (1994). However, for this new $\mathrm{H} \alpha$ visualization method we evaluated these parameters for a broader range of temperatures using the most advanced 1D non-LTE radiative transfer models recently implemented by Heinzel et al. (2014). The results are given in Table 1 for three representative altitudes of 10000,20000 , and $30000 \mathrm{~km}$ and for a nominal slab thickness of $500 \mathrm{~km}$. For the isothermal-isobaric slabs of Heinzel et al. (2014) that are not optically thick in $\mathrm{H} \alpha$, the atomic level populations and the electron densities are almost constant inside the slabs (Gouttebroze et al. 1993), and we took the values at the slab center to tabulate $i$ and $f$. For the same altitudes we also determined average values of the $\mathrm{H} \alpha$ line source function $S$ as $3.22 \times 10^{-6}, 2.98 \times 10^{-6}$, and $2.80 \times 10^{-6} \mathrm{erg} \mathrm{s}^{-1} \mathrm{~cm}^{-2} \mathrm{sr}^{-1} \mathrm{~Hz}^{-1}$, respectively. The values of $S$ and $f$ can be approximately scaled in altitude by the ratio of the dilution factors, as these quantities are proportional to the dilution factor by their very nature. The ionization degree $i$ varies much less with the altitude and is not directly related to the dilution factor $W$.

\section{Recipes for prominence or filament visualizations}

To visualize the whole-prominence magnetic field configuration containing many magnetic dips filled with the plasma having a
Table 1. Values of the ionization degree $i$ and the parameter $f$ for various values of the temperature (in $\mathrm{K}$ ) and gas pressure (in dyn $\mathrm{cm}^{-2}$ ).

\begin{tabular}{ccccccc}
\hline \hline \multicolumn{7}{c}{ Altitude $10000 \mathrm{~km}$} \\
\hline \multirow{5}{*}{$i$} & $p_{\mathrm{g}}$ & 0.01 & 0.02 & 0.05 & 0.10 & 0.20 \\
\hline & 6000 & 0.74 & 0.62 & 0.44 & 0.31 & 0.20 \\
& 8000 & 0.83 & 0.72 & 0.55 & 0.44 & 0.35 \\
& 10000 & 0.87 & 0.79 & 0.70 & 0.69 & 0.73 \\
& 12000 & 0.91 & 0.85 & 0.82 & 0.85 & 0.89 \\
& 14000 & 0.93 & 0.89 & 0.89 & 0.92 & 0.94 \\
\hline \multirow{5}{*}{$f\left[10^{16}\right]$} & 6000 & 5.0 & 4.6 & 4.2 & 4.0 & 4.0 \\
& 8000 & 6.7 & 5.8 & 5.0 & 4.8 & 4.7 \\
& 10000 & 8.1 & 6.8 & 5.3 & 5.1 & 5.3 \\
& 12000 & 9.1 & 7.0 & 5.0 & 4.9 & 5.2 \\
& 14000 & 9.8 & 7.1 & 4.9 & 4.8 & 5.0 \\
\hline
\end{tabular}

\begin{tabular}{|c|c|c|c|c|c|c|}
\hline \multicolumn{7}{|c|}{ Altitude $20000 \mathrm{~km}$} \\
\hline & $T_{T} \quad p_{\mathrm{g}}$ & 0.01 & 0.02 & 0.05 & 0.10 & 0.20 \\
\hline \multirow{5}{*}{$i$} & 6000 & 0.73 & 0.60 & 0.41 & 0.29 & 0.18 \\
\hline & 8000 & 0.81 & 0.70 & 0.52 & 0.41 & 0.33 \\
\hline & 10000 & 0.86 & 0.78 & 0.68 & 0.68 & 0.72 \\
\hline & 12000 & 0.90 & 0.84 & 0.81 & 0.84 & 0.88 \\
\hline & 14000 & 0.92 & 0.88 & 0.89 & 0.91 & 0.94 \\
\hline \multirow{5}{*}{$f\left[10^{16}\right]$} & 6000 & 4.7 & 4.2 & 3.8 & 3.7 & 3.6 \\
\hline & 8000 & 6.3 & 5.4 & 4.6 & 4.4 & 4.3 \\
\hline & 10000 & 7.6 & 6.3 & 4.8 & 4.7 & 4.9 \\
\hline & 12000 & 8.6 & 6.5 & 4.6 & 4.5 & 4.8 \\
\hline & 14000 & 9.2 & 6.4 & 4.5 & 4.4 & 4.6 \\
\hline
\end{tabular}

\begin{tabular}{|c|c|c|c|c|c|c|}
\hline \multicolumn{7}{|c|}{ Altitude $30000 \mathrm{~km}$} \\
\hline & ${ }_{T} p_{\mathrm{g}}$ & 0.01 & 0.02 & 0.05 & 0.10 & 0.20 \\
\hline \multirow{5}{*}{$i$} & 6000 & 0.71 & 0.58 & 0.39 & 0.27 & 0.17 \\
\hline & 8000 & 0.80 & 0.68 & 0.50 & 0.39 & 0.32 \\
\hline & 10000 & 0.85 & 0.76 & 0.66 & 0.67 & 0.71 \\
\hline & 12000 & 0.89 & 0.83 & 0.81 & 0.84 & 0.88 \\
\hline & 14000 & 0.92 & 0.88 & 0.88 & 0.91 & 0.93 \\
\hline \multirow{5}{*}{$f\left[10^{16}\right]$} & 6000 & 4.5 & 4.0 & 3.5 & 3.4 & 3.4 \\
\hline & 8000 & 6.1 & 5.1 & 4.3 & 4.0 & 4.0 \\
\hline & 10000 & 7.4 & 6.0 & 4.5 & 4.3 & 4.5 \\
\hline & 12000 & 8.3 & 6.1 & 4.2 & 4.2 & 4.5 \\
\hline & 14000 & 8.9 & 6.0 & 4.2 & 4.1 & 4.3 \\
\hline
\end{tabular}

given temperature and pressure distribution, we proceed in the following way. First, we compute the LOS optical thickness

$\tau_{v}=\int_{\operatorname{LOS}} \kappa(v, l) \mathrm{d} l$,

where the line absorption coefficient $\kappa(v, l)$ is given by Eq. (4). For a given height above the solar surface, we can interpolate the value of the $\mathrm{H} \alpha$ source function given in Sect. 2.3. The values of $i$ and $f$ entering the evaluation of the absorption coefficient are obtained from Table 1 for the local values of $T$ and $p_{\mathrm{g}}$ along the LOS. Then Eq. (3) is used to compute the synthetic line profile for each LOS (a pixel in the simulated image). To obtain the synthetic image for a specific $\mathrm{H} \alpha$ filter, one has to multiply the emission line profile by the filter response function and integrate over the wavelength range of the filter sensitivity. We note that for $\tau_{v}>1$, the line intensity will saturate to the value of the source function, as evident from Eq. (3).

The same procedure can be also applied to visualize filaments viewed against the solar disk. However, in this case, the 
background disk radiation attenuated by the filament must be added, that is,

$I(v)=S\left(1-\mathrm{e}^{-\tau_{v}}\right)+I_{\mathrm{bgr}} \mathrm{e}^{-\tau_{v}}$.

Depending on the position of the filament on the solar disk, the background $\mathrm{H} \alpha$ intensity $I_{\text {bgr }}$ can be obtained from David (1961), for instance, and using the limb-darkened continuum intensity in the vicinity of the $\mathrm{H} \alpha$ line (Allen 1976). We note that for filaments, $S$ varies with height along the LOS (see Sect. 2.3), which might require a more accurate solution.

The visualization method presented here can also accommodate LOS velocities on the order of $10 \mathrm{~km} \mathrm{~s}^{-1}$. Such velocities are typically encountered in quiescent prominences, see Gunár et al. (2012), for example. The source function and the ionization degree are not significantly affected by such velocities, which allows us to use the same tabulated values of $S, f$, and $i$. These velocities therefore only affect the absorption coefficient $\kappa(v, l)$. Taking into account a local Doppler shift $\Delta v^{\prime}(l)$ due to the local LOS velocity, we can modify Eq. (4) as

$\kappa(v, l)=1.7 \times 10^{-2} n_{2} \phi\left(v-\Delta v^{\prime}(l)\right)$.

\subsection{Tests of the method}

To demonstrate the accuracy of our $\mathrm{H} \alpha$ visualization method, we performed several tests comparing the $\mathrm{H} \alpha$ line intensities obtained by $1 \mathrm{D}$ and $2 \mathrm{D}$ non-LTE radiative transfer models with the results of the fast approximate $\mathrm{H} \alpha$ synthesis.

In the first test we used the series of 1D non-LTE isothermalisobaric models of Heinzel et al. (2014). We compared the $\mathrm{H} \alpha$ line-center intensities obtained by the models with the intensities computed by the $\mathrm{H} \alpha$ synthesis when using the interpolation of parameters $i$ and $f$ from the tables provided in this paper and when using representative fixed values of parameters $i$ and $f$ for all models. All results are valid for the altitude of $10000 \mathrm{~km}$. In Fig. 1 (top) we show the scatterplot of the $\mathrm{H} \alpha$ line-center intensities obtained by 1D non-LTE computations ( $x$-axis) and our approximate synthesis using $i$ and $f$ interpolation ( $y$-axis). The bottom part of Fig. 1 shows the same scatterplot, but using the approximate synthesis with representative values of $i=0.35$ and $f=4.7 \times 10^{16} \mathrm{~cm}^{-3}$. It is evident that the intensities obtained by the approximate synthesis with $i$ and $f$ interpolation agree very well with the intensities obtained by exact 1D non-LTE computations. The approximate synthesis with selected representative $i$ and $f$ values also produces results that agree reasonably well with the exact solutions.

In the second test we used the $2 \mathrm{D}$ temperature and gas pressure distribution inside the force-free magnetic dip named DEEP_DIP presented in Gunár et al. (2013), see Fig. 6 therein. We scanned its 2D structure along its length with the LOS perpendicular to the $x$-axis and at each position computed the $\mathrm{H} \alpha$ line-center intensity and optical thickness using the approximate $\mathrm{H} \alpha$ synthesis with the interpolation of $i$ and $f$. We compared this with the results of the $2 \mathrm{D}$ non-LTE radiative transfer modeling reported by Gunár et al. (2013). In the top row of Fig. 2 we plot the optical thickness (left) and the intensity (right) at the $\mathrm{H} \alpha$ line center along the length of the magnetic dip. Solid lines represent the full 2D non-LTE radiative transfer calculations and dashed-dotted lines show the approximate $\mathrm{H} \alpha$ synthesis. In the bottom row of Fig. 2 we show the same, but for the 2D fine-structure thread model MoDEL_DEM presented by Gunár et al. (2011), see Fig. 6 therein. Again, we plot the optical thickness (left) and the intensity (right) using solid lines for
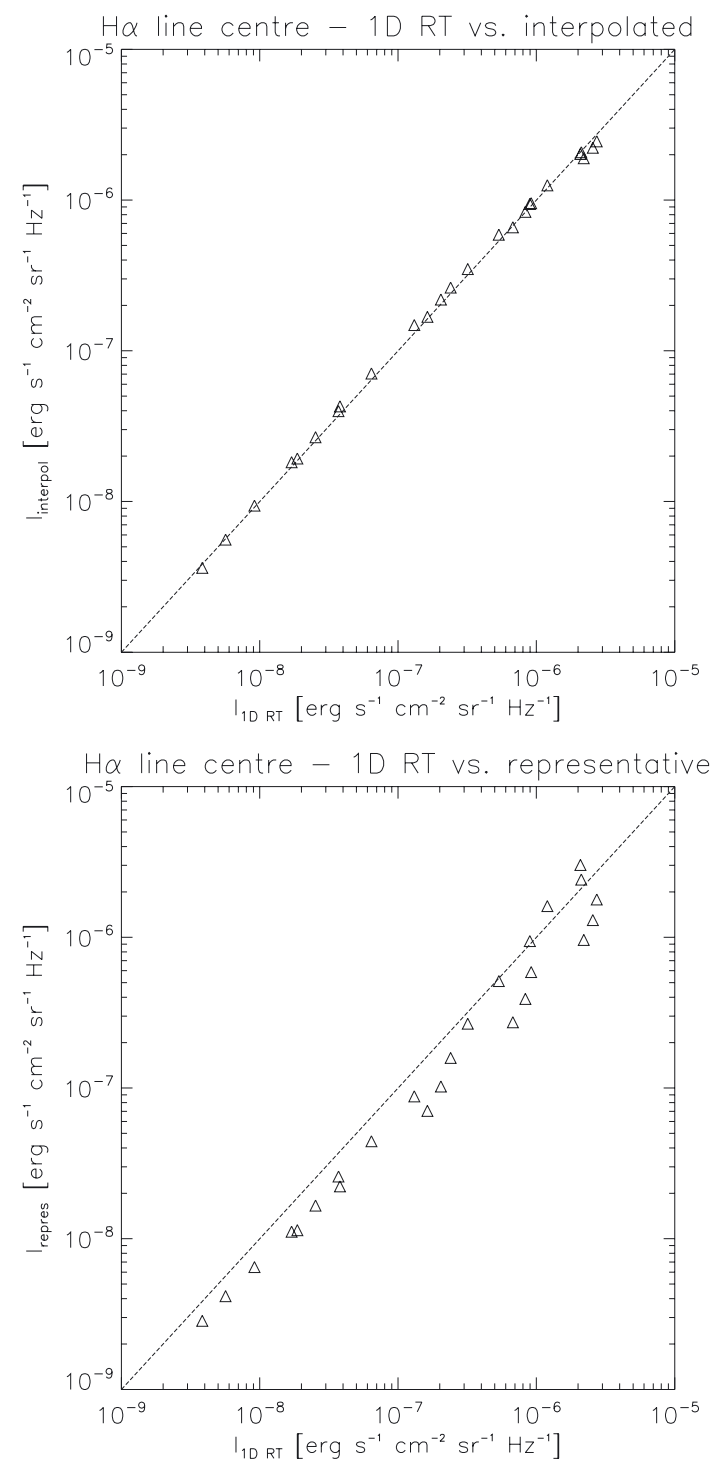

Fig. 1. Scatterplots of the $\mathrm{H} \alpha$ line center intensities obtained by 1 D non-LTE computations ( $x$-axes) and by approximate synthesis using $i$ and $f$ interpolation (top) and using fixed representative $i$ and $f$ values (bottom). Dashed lines correspond to the precise match with the intensities obtained by $1 \mathrm{D}$ non-LTE computations.

the full 2D non-LTE radiative transfer calculations and dasheddotted lines for the approximate $\mathrm{H} \alpha$ synthesis. The plots in Fig. 2 show that the approximate synthesis is also able to reproduce the optical thickness and the intensity of the $\mathrm{H} \alpha$ line center as derived by more complex 2D non-LTE calculations reasonably well. This represents an important confirmation of the reliability of the approximate synthesis method that is based on the 1D prominence fine-structure modeling (Heinzel et al. 2014). A mild influence of the $2 \mathrm{D}$ radiative transfer effects is apparent in the first row of Fig. 2, where the discrepancy at the sides of the structure (wings of the plotted $\tau$ and $I$ profiles) can be attributed to the influence of the incident radiation penetrating the $2 \mathrm{D}$ structure from the sides. The approximate 1D synthesis cannot account for these boundary effects. The discrepancy between the approximate 1D synthesis and the 2D non-LTE calculations shown in the second row of Fig. 2 might also be caused by the $2 \mathrm{D}$ radiative transfer effects. In this case, the approximate synthesis underestimates the $\mathrm{H} \alpha$ line center optical thickness and intensity in the middle of the structure while correctly 
P. Heinzel et al.: $\mathrm{H} \alpha$ visualization of prominence fine structures
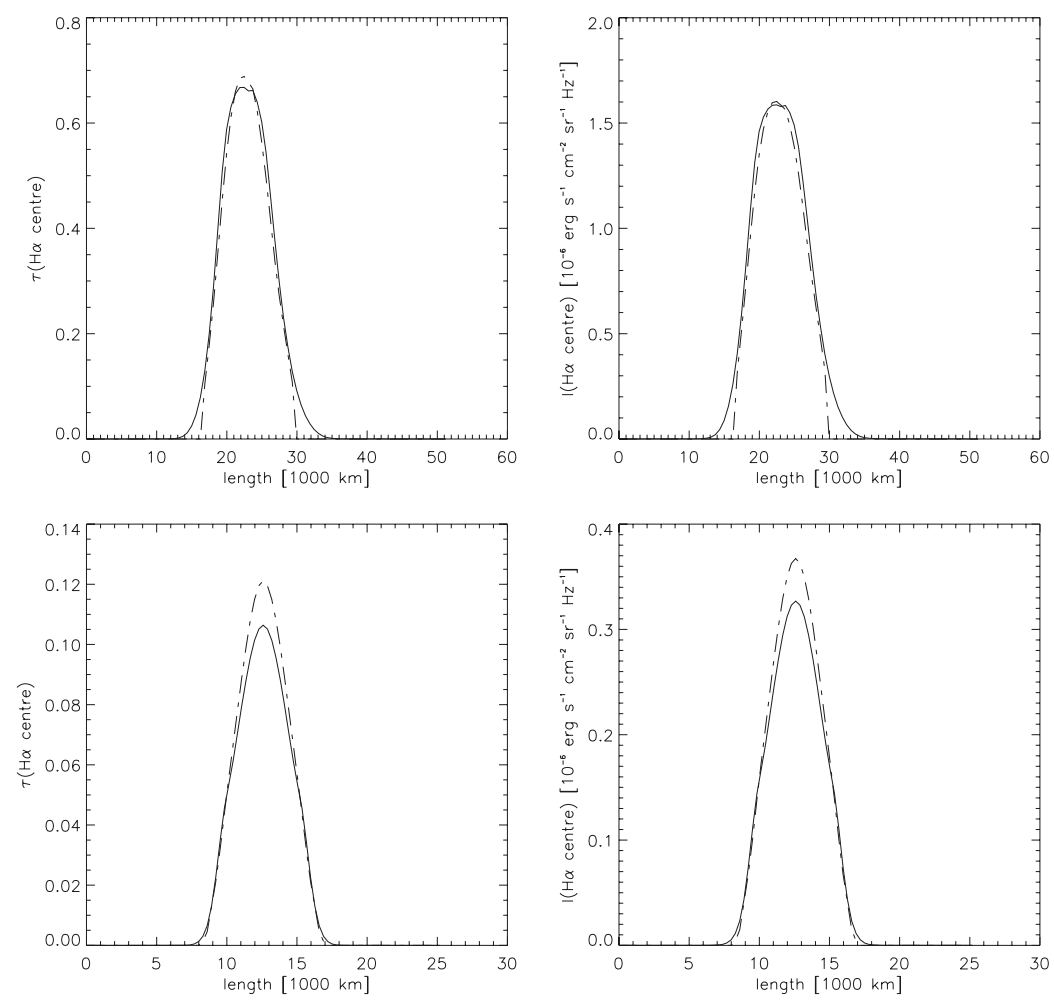

Fig. 2. Optical thickness (left column) and intensity (right column) at the $\mathrm{H} \alpha$ line center plotted along the length of the 2D plasma structure of the DEEP_DIP (upper row) and the MODEL_DEM (lower row). Solid lines represent the 2D non-LTE radiative transfer calculations, while the dash-dotted lines show the results of the approximate $\mathrm{H} \alpha$ synthesis with the interpolation of $i$ and $f$.

representing its sides. We note that our test-cases represent different 2D prominence fine-structure models - the first based on the force-free magnetic dips and the second using gravityinduced dips. We used them to demonstrate the ability of the approximate $\mathrm{H} \alpha$ synthesis method to reproduce results for a wide variety of models, but it is not our aim to compare these models with each other.

\section{Sample image of the prominence magnetic dip}

To illustrate the application of the approximate $\mathrm{H} \alpha$ line synthesis method presented here, we visualized two force-free magnetic dips filled with prominence plasma. The magnetic field configuration of these dips was obtained by the 3D nonlinear force-free field simulations of the whole prominence magnetic structure by Mackay \& van Ballegooijen (2009). These dips were filled with prominence plasma in the hydrostatic equilibrium according to the method of Gunár et al. (2013). One of the visualized dips is the DEEP_DIP, representing a prominence fine structure with higher gas pressure in its center due to its significant depth, see Gunár et al. (2013). The other dip is shallower (but not the SHALLOW_DIP of Gunár et al. 2013), resulting in a much lower central gas pressure. It is also more extended along the magnetic field, about $65000 \mathrm{~km}$ compared to the $50000 \mathrm{~km}$ of the DEEP_DIP. Both dips simulate prominence fine structures with an assumed cross-section of $1000 \mathrm{~km}$. In Fig. 3 we show a synthetic image in the $\mathrm{H} \alpha$ line center where the DEEP_DIP is placed above the shallower dip. The DEEP_DIP appears much brighter due to its higher central gas pressure. Moreover, it also appears somewhat longer than the (in reality more extended) shallower dip. This can be attributed to the $\mathrm{H} \alpha$ visibility of the prominence material.

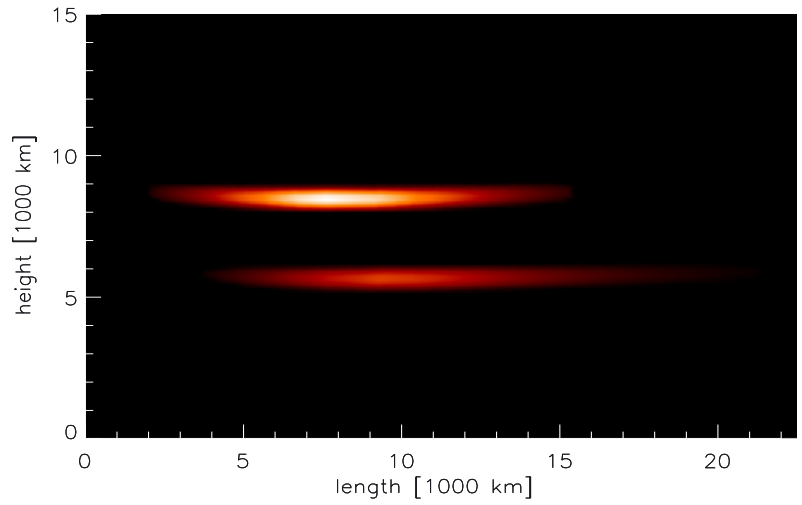

Fig. 3. Synthetic image in the $\mathrm{H} \alpha$ line center of the DEEP_DIP (upper structure) and a shallower dip (lower structure).

\section{Discussion and conclusions}

As mentioned above, our approximate $\mathrm{H} \alpha$ visualization method assumes that the individual fine-structure elements do not interact radiatively, and thus each of them is only illuminated by the incident solar radiation. In this case, their $\mathrm{H} \alpha$ line source function can be determined using the non-LTE radiative transfer model (1D slabs in our case) for individual fine structures. For these 1D models we considered rather narrow slabs of $500 \mathrm{~km}$ thickness to obtain the values of $S, i$, and $f$ tabulated in Sect. 2.3. These values are taken at the slab center but result from the radiative transfer within the whole slab. However, in the approximate $\mathrm{H} \alpha$ synthesis method we considered them as local values. This assumption introduces certain errors in the estimates of the true local values of these parameters. However, the tests 
presented in Sect. 3.1 show that our approximate $\mathrm{H} \alpha$ visualization method is sufficiently accurate. We note that this accuracy would break down in an extreme case when all prominence fine structures were densely packed close to each other within a large prominence. This scenario may be simulated by a large homogeneous slab that is only illuminated at its two boundaries. In this case, the depth-dependent behavior of the source function will be different, reflecting the actual conditions within the (optically thick) slab - see Gouttebroze et al. (1993).

We have demonstrated that our fast approximate radiative transfer method for vizualising the $\mathrm{H} \alpha$ prominence provides realistic synthetic $\mathrm{H} \alpha$ intensities that are useful for a reliable visualization of prominences and filaments. Such synthetic highresolution images of whole prominences/filaments are quantitatively accurate enough and thus can be used for a direct comparison with radiometrically calibrated observations.

Acknowledgements. P.H. acknowledges the support from grant 209/12/0906 of the Grant Agency of the Czech Republic. S.G. acknowledges support from the European Commission via the Marie Curie Actions - Intra-European Fellowships Project No. 328138. P.H. and S.G. acknowledge support from project RVO:67985815 of the Astronomical Institute of the Czech Academy of Sciences and from the MPA Garching. U.A. thanks for the support from the Astronomical Institute of the Czech Academy of Sciences.

\section{References}

Allen, C. W. 1976, Astrophysical Quantities (London: The Athlone Press) Aulanier, G., \& Démoulin, P. 1998, A\&A, 329, 1125
David, K.-H. 1961, Z. Astrophys., 53, 37

Dudík, J., Aulanier, G., Schmieder, B., Bommier, V., \& Roudier, T. 2008, Sol. Phys., 248, 29

Engvold, O., Hirayama, T., Leroy, J. L., Priest, E. R., \& Tandberg-Hanssen, E. 1990, in Dynamics of Quiescent Prominences, eds. V. Ruzdjak \& E. Tandberg-Hanssen (New York: Springer Verlag), IAU Colloq., 363, 294 Gouttebroze, P., Heinzel, P., \& Vial, J. C. 1993, A\&AS, 99, 513

Gunár, S. 2014, in IAU Symp. 300, eds. B. Schmieder, J.-M. Malherbe, \& S. T. Wu, 59

Gunár, S., \& Mackay, D. H. 2015, ApJ, 803, 64

Gunár, S., Parenti, S., Anzer, U., Heinzel, P., \& Vial, J.-C. 2011, A\&A, 535, A122

Gunár, S., Mein, P., Schmieder, B., Heinzel, P., \& Mein, N. 2012, A\&A, 543, A93

Gunár, S., Mackay, D. H., Anzer, U., \& Heinzel, P. 2013, A\&A, 551, A3

Heinzel, P. 2015, in Astrophys. Space Sci. Lib. 415, eds. J.-C. Vial, \& O. Engvold, 103

Heinzel, P., \& Anzer, U. 2001, A\&A, 375, 1082

Heinzel, P., Gouttebroze, P., \& Vial, J.-C. 1994, A\&A, 292, 656

Heinzel, P., Vial, J.-C., \& Anzer, U. 2014, A\&A, 564, A132

Jejčič, S., \& Heinzel, P. 2009, Sol. Phys., 254, 89

Karpen, J. T., Antiochos, S. K., \& Klimchuk, J. A. 2006, ApJ, 637, 531

Labrosse, N., Heinzel, P., Vial, J., et al. 2010, Space Sci. Rev., 151, 243

Landman, D. A., \& Mongillo, M. 1979, ApJ, 230, 581

Leenaarts, J., Carlsson, M., \& Rouppe van der Voort, L. 2012, ApJ, 749, 136

Leenaarts, J., Carlsson, M., \& Rouppe van der Voort, L. 2015, ApJ, 802, 136

Luna, M., Karpen, J. T., \& DeVore, C. R. 2012, ApJ, 746, 30

Mackay, D. H., \& van Ballegooijen, A. A. 2009, Sol. Phys., 260, 321

Mackay, D. H., Karpen, J. T., Ballester, J. L., Schmieder, B., \& Aulanier, G. 2010, Space Sci. Rev., 151, 333

Su, Y., \& van Ballegooijen, A. 2012, ApJ, 757, 168

van Ballegooijen, A. A., \& Su, Y. 2014, in IAU Symp. 300, eds. B. Schmieder, J.-M. Malherbe, \& S. T. Wu, 127 